\title{
Floristic Diversity of Thevarmala Sacred Grove in Western Ghats, Kerala, India
}

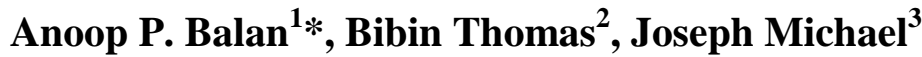 \\ ${ }^{1}$ Indian Cardamom Research Institute, Myladumpara, Kailasanadu P.O., Kerala, India \\ ${ }^{2,3}$ Department of Botany, St. Dominic's College, Kanjirapally, Kerala, India
}

\begin{abstract}
A study on the flowering plant diversity of Thevarmala sacred grove in the Western Ghats of Kerala region was carried out during the period 2015-2016. During the study a total of 152 species of flowering plants belong to 136 genera under 50 families were documented. Plants in all life forms, viz. herbs (56 species), shrubs (64 species) and trees (32 species) are well represented in the grove. Fabaceae is the dominant family with 15 species in 12 genera followed by Euphorbiaceae and Rubiaceae. As many as 17 families are represented by single species in each. Out of the 152 species 18 are rare endemics especially confined to the southern Western Ghats. About $62 \%$ of the documented plants are having economic importance as medicine (76 species), food (8 species), timber (6 species), and fodder (3 species) or as source of commercially useful fibres and resins. 19 species documented in the present study are new record to the Kottayam district of Kerala state. The present conservation status of this sacred grove and future threats are also discussed in this paper.
\end{abstract}

Keywords: Sacred groves, Western Ghats, Kerala, Floristic diversity, Conservation.

\section{INTRODUCTION}

Sacred groves are sanctified patches of native vegetation traditionally been protected on the ground of religious beliefs and are generally dedicated to different gods, goddesses, spirits, serpents, etc. These are the remnants of a rich indigenous vegetation that has existed in an area in the distant past and are survived the axe of development simply on grounds of religious beliefs. Each sacred grow has a deity and people are afraid to anger the deities when damaging the vegetation or overexploiting other resources of sacred groves as it is strongly believed that the deities will punish by bringing illness, deaths or misfortune. Religious sentiments thus played a critical role in the conservation of these natural treasures for centuries.

As described by Vartak (1983), sacred groves are natural museums of living giant trees, treasure house of rare, endemic and endangered species, dispensary of medicinal plants, recreation centre for urban life, garden for botanists, gene bank of economic species, paradise for nature lovers and laboratory for environmentalists. They are considered as repository of local biodiversity. The sacred groves play an important role in ecosystem services by providing clean air, soil and water, conservation of flora and fauna, temperature control and conservation of traditional knowledge. Being a biotype in a rural landscape, the sacred groves performs a critical role in the maintenance of ecological and hydrological balance of an area, and also help to compensate for carbon emissions of polluting industries and thereby offers economic benefits to the communities besides other ecological benefits (Chandrashekara, 2011).

Sacred groves are found in all continents except Antartica with varied religions and forms of social and economic organizations (Hughes \& Chandran, 1998). In India the sacred groves are found scattered all over the country especially in the Khasi and Jaintia hills of Northeastern Himalayas, Aravalli Hills, Central India and Western Ghats. They are known under different names in different states like Dev van in Himachal Pradesh, Orans in Rajasthan, Sarnas in Bihar, Lai Umang in Manipur, Ki Law Kyntang in Meghalaya, Dev or Sarna in Madhya Pradesh, Deovani, Devrai or Devrahati in Maharashtra, Devarabana, Devarakadu or Pavitravana in Karnataka, Kavu or Kovilkadu in Tamil Nadu, and Kavu in Kerala (Murugan et al., 2008).

Sacred groves are seen throughout Kerala from coastal areas to the ghats and are having different cultural practices and belief systems. The area of sacred groves varies from few trees to several hectares. In Kerala, the sacred groves are mostly distributed along the plains of northern and southern 
Kerala and are comparatively well studied (Induchoodan, 1998, Jayarajan, 2004; Chandrashekara, 2011; Sreevidhya, 2014). However, the sacred groves in central Kerala, especially in Kottayam and Idukki districts are poorly explored. Haritha et al. (2014 \& 2015) studied the floristic wealth of some selected sacred groves in Kottayam district. However, the studied groves are located in the plains and are representative of low land vegetation of Kottayam district. In this scenario, the present investigation on the floristic diversity of Thevarmala sacred grove is of special relevance, since this sacred grove represents the few sacred groves in Kerala above $400 \mathrm{~m}$ altitude with unique Western Ghats floristic composition. The evergreen forests in the high ranges of Kottayam district are widely cleared a century ago for the establishment of Rubber plantations and Thevarmala sacred grove has become a refuge for some rare endemic and medicinally useful plants in that area.

\subsection{Study Area}

The study area, Thevarmala sacred grove is located in central Kerala, near Kayyur, in the Bharananganam panchayat of Kottayam revenue district, lies between $9^{0} 75^{\prime}$ North latitude and $76^{0}$ 70' East longitude (Fig. 1.a.). The grove is situated on the top of a small hill called Thevarmala, at $450-480 \mathrm{~m}$ altitude above the mean sea level and covers an area of 15 acres of land. The grove is associated with a temple dedicated to Lord Sankaranarayana and Pandavas, since the origin of the grove is related to the epic Mahabharatha. The grove is managed by three Nair families namely, Kulappuram, Chooramala and Machukad. Womens are not allowed to enter in to the grove and temple.
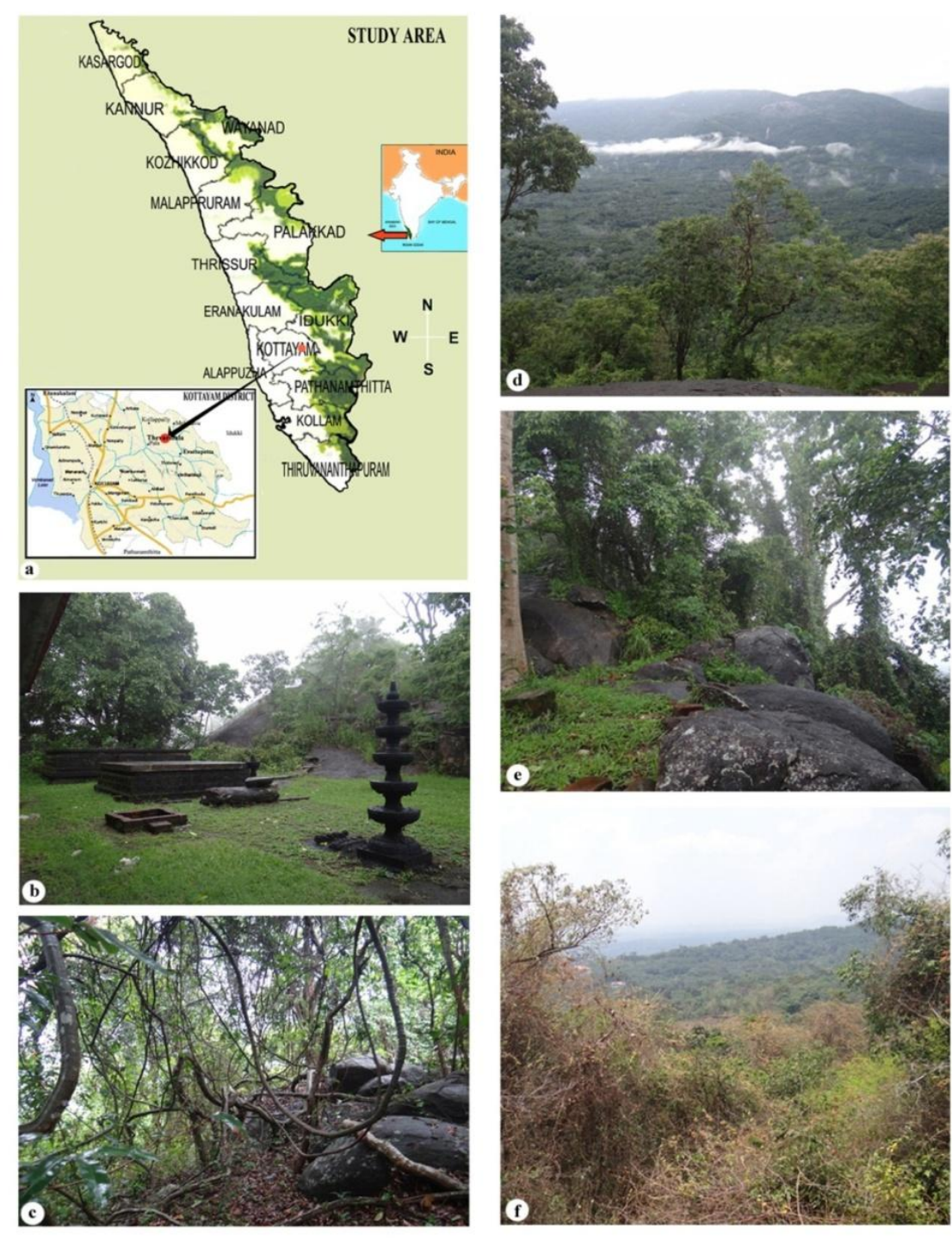

Figure 1. a. Location of Thevarmala sacred grove; $b-f$. Vegetation of Thevarmala sacred grove. 


\subsection{Climate and vegetation}

This sacred grove is located in the border of Kottayam and Idukki district in southern Western Ghats and receives comparatively higher rate of south-west and north-east monsoon. The mean annual rainfall is $2700 \mathrm{~mm}$. The mean annual temperature varies from $25^{\circ} \mathrm{C}$ to $31^{\circ} \mathrm{C}$. The temperature drops to $18^{0} \mathrm{C}-20^{\circ} \mathrm{C}$ during winter and raises to $35^{\circ} \mathrm{C}-38^{0} \mathrm{C}$ in summer months. The mean relative humidity varies from 80-90 \% during rainy seasons and lowers to $65-70 \%$ in summer months.

The natural vegetation of the sacred grove is mixed deciduous type (Fig. 1.b.-f.). During the rainy season the canopy looks similar to that of semi-evergreen forests and during dry season some of the trees shed their leaves and revealed its deciduous nature. Albizia odoratissima (L. f.) Benth., Ceiba pentandra (L.) Gaertn., Dalbergia latifolia Roxb., Pterocarpus marsupium Roxb., Strychnos nuxvomica L., Terminalia paniculata Roth, Vitex altissima L. f., Wrightia tinctoria (Roxb.) R. Br. etc. are the dominating trees. Undergrowth is dominated by Allophylus cobbe (L.) Raeusch., Breynea retusa (Dennst.) Alston, Canthium coromandelicum (Burm. f.) Alston, Chassalia curviflora (Wall. ex Kurz) Thw., Psilanthus travancorensis (Wight \& Arn.) Leroy etc. Aganosma cymosa (Roxb.) G. Don, Dioscorea oppositifolia L., Jasminum coarctatum Roxb., Spatholobus parviflorus (Roxb. ex DC.) O. Ktze., Strychnos minor Dennst., Toxocarpus kleinii Wight \& Arn., Zehneria maysorensis (Wight \& Arn.) Arn. etc. are the major climbers.

\section{Materials AND Methods}

Extensive floristic explorations were conducted in Thevarmala sacred grove during the period 20152016 in different seasons. Specimens of each taxon were collected and herbarium sheets were prepared as per the standard practices. Plants are identified with the help of regional floras like The Flora of Presidency of Madras (Gamble, 1915-1936), Flowering Plants of Travancore (Ram Rao, 1914.) etc. and also with the help of experts from Kerala Forest Research Institute, Peechi. Nomenclature and distribution details of each taxon are based on Sasidharan (2004) and Nair et al., (2014). The socio-cultural history of the grove is collected from the senior members of the governing families.

\section{RESUlTS \& Discussion}

The present study was undertaken to assess the angiosperm plant diversity of Thevarmala sacred grove, Kottayam District, Kerala. This sacred grove is rather rich in its floristic composition. Mixtures of both evergreen and deciduous elements are found in the grove. A total of 152 species of flowering plants belong to 136 genera under 50 families were documented (Table 1). Dicots are dominating with 129 species in 41 families; meanwhile monocots are represented by 23 species in 22 genera under 9 families. In dicots, the group polypetalae is represented by 48 species ( 45 genera under 20 families), gamopetalae by 54 species (48 genera under 14 families) and monochlamydeae by 27 species (21 genera under 7 families). Details are illustrated in Fig.2.

Table1. List of flowering plants in Thevarmala sacred grove.

\begin{tabular}{|l|l|l|}
\hline & Name of the plant & Utility \\
\hline & 1. ACANTHACEAE & \\
\hline 1 & Andrographis atropurpurea (Dennst.) Alston & \\
\hline 2 & Justicia diffusa Willd. & \\
\hline 3 & Justicia japonica Thunb. & Medicine (Ambasta, 1986) \\
\hline 4 & Phaulopsis imbricata (Forssk.) Sweet. & \\
\hline & 2. AMARANTHACEAE & Medicine (Vijaya Raghavan, 2011) \\
\hline 5 & Alternanthera brasiliana ( L. ) Kuntze & \\
\hline 6 & Cyathula prostrata (L.) Blume & Medicine (Nambiar et al., 1985) \\
\hline 7 & 3. ANACARDIACEAE & Medicine (Kirtikar \& Basu, 1918) \\
\hline 8 & Holigarna arnottiana Hook. f. & \\
\hline 9 & Mangifera indica L. & \\
\hline & Solenocarpus indicus Wight \& Arn. & \\
\hline 10 & 4. APOCYNACEAE & Medicine (Vijaya Raghavan, 2011) \\
\hline 11 & Aganosma cymosa (Roxb.) G. Don & Medicine (Nambiar et al., 1985) \\
\hline 12 & Alstonia scholaris (L.) R. Br. & \\
\hline
\end{tabular}


Anoop P. Balan et al.

\begin{tabular}{|c|c|c|}
\hline 13 & Ichnocarpus frutescens (L.) R. Br. & Medicine (Nambiar et al., 1985) \\
\hline 14 & Tabernaemontana alternifolia $\mathrm{L}$. & Medicine (Nambiar et al., 1985) \\
\hline \multirow[t]{2}{*}{15} & Wrightia tinctoria (Roxb.) R. Br. & Medicine (Nambiar et al., 1985) \\
\hline & 5. ARACACEAE & \\
\hline 16 & Calamus rotang $\mathrm{L}$. & \\
\hline \multirow[t]{2}{*}{17} & Caryota urens $\mathrm{L}$. & Medicine (Nambiar et al., 1985) \\
\hline & 6. ARACEAE & \\
\hline 18 & Colocasia esculenta (L.) Schott & $\begin{array}{l}\text { Medicine (Nambiar et al., 1985) } \\
\text { Food (Ambasta, 1986) }\end{array}$ \\
\hline \multirow[t]{2}{*}{19} & Pothos scandens L. & Medicine (Parrota, 2001) \\
\hline & 7. ARALIACEAE & \\
\hline \multirow[t]{2}{*}{20} & Schefflera venulosa (Wight \& Arn.) Harms & \\
\hline & 8. ARISTOLOCHIACEAE & \\
\hline 21 & Aristolochia indica $\mathrm{L}$. & Medicine (Nambiar et al., 1985) \\
\hline \multirow[t]{2}{*}{22} & Thottea duchartrei Sivar. & \\
\hline & 9. ASCLEPIADACEAE & \\
\hline 23 & Cosmostigma racemosum (Roxb.) Wight & Medicine (Kirtikar \& Basu, 1918) \\
\hline 24 & Gymnema sylvestre (Retz.) R. Br. ex Schult. & Medicine (Kirtikar \& Basu, 1918) \\
\hline \multirow[t]{2}{*}{25} & Toxocarpus kleinii Wight \& Arn. & \\
\hline & 10. ASTERACEAE & \\
\hline 26 & Ageratum conyzoides $\mathrm{L}$. & Medicine (Kirtikar \& Basu, 1918) \\
\hline 27 & Chromolaena odorata (L.) King \& Robins. & \\
\hline 28 & Crassocephalum crepidioides (Benth.) S. Moore & Medicine (Ambasta, 1986) \\
\hline 29 & Elephantopus scaber L. & Medicine (Parrota, 2001) \\
\hline 30 & Mikania micrantha Kunth & \\
\hline 31 & Synedrella nodiflora (L.) Gaertn. & \\
\hline 32 & Tridax procumbens $\mathrm{L}$. & Medicine (Ambasta, 1986) \\
\hline \multirow[t]{2}{*}{33} & Vernonia cinerea (L.) Less. & Medicine (Kirtikar \& Basu, 1918) \\
\hline & 11. BALSAMINACEAE & \\
\hline \multirow[t]{2}{*}{34} & Impatiens flaccida Arn. & \\
\hline & 12. BOMBACACEAE & \\
\hline \multirow[t]{2}{*}{35} & Ceiba pentandra (L.) Gaertn. & $\begin{array}{l}\text { Medicine (Kirtikar \& Basu, 1918) } \\
\text { Fibre (Ambasta, 1986) }\end{array}$ \\
\hline & 13. CAPPARACEAE & \\
\hline \multirow[t]{2}{*}{36} & Cleome monophylla L. & \\
\hline & 14. COMBRETACEAE & \\
\hline 37 & Calycopteris floribunda Lam. & Medicine (Nambiar et al., 1985) \\
\hline \multirow[t]{2}{*}{38} & Terminalia paniculata Roth & $\begin{array}{l}\text { Medicine (Kirtikar \& Basu, 1918) } \\
\text { Timber (Rama Rao, 1914) }\end{array}$ \\
\hline & 15. COMMELINACEAE & \\
\hline 39 & Commelina ensifolia $\mathrm{R} . \mathrm{Br}$. & \\
\hline \multirow[t]{2}{*}{40} & Cyanotis pilosa Schult. \& Schult. f. & \\
\hline & 16. CONVOLVULACEAE & \\
\hline 41 & Argyreia hirsuta Wight \& Arn. & \\
\hline 42 & Hewittia malabarica (L.) Suresh & \\
\hline 43 & Ipomoea mauritiana Jacq. & \\
\hline 44 & Ipomoea triloba $\mathrm{L}$. & \\
\hline \multirow[t]{2}{*}{45} & Xenostegia tridentata (L.) Austin \& Staples & \\
\hline & 17. CUCURBITACEAE & \\
\hline \multirow[t]{2}{*}{46} & Zehneria maysorensis (Wight \& Arn.) Arn. & \\
\hline & 18. CYPERACEAE & \\
\hline \multirow[t]{2}{*}{47} & Cyperus dubius Rottb. & \\
\hline & 19. DIOSCOREACEAE & \\
\hline 48 & Dioscorea bulbifera L. & $\begin{array}{l}\text { Medicine (Kirtikar \& Basu, 1918) } \\
\text { Food (Rama Rao, 1914) }\end{array}$ \\
\hline
\end{tabular}


Floristic Diversity of Thevarmala Sacred Grove in Western Ghats, Kerala, India

\begin{tabular}{|c|c|c|}
\hline 49 & Dioscorea oppositifolia L. & $\begin{array}{l}\text { Medicine (Kirtikar \& Basu, 1918) } \\
\text { Food (Rama Rao, 1914) }\end{array}$ \\
\hline & 20. DIPTEROCARPACEAE & \\
\hline \multirow[t]{2}{*}{50} & Vateria indica $\mathrm{L}$. & $\begin{array}{l}\text { Medicine (Kirtikar \& Basu, 1918) } \\
\text { Food, Soft wood,Resin (Ambasta, } \\
\text { 1986) }\end{array}$ \\
\hline & 21. EUPHORBIACEAE & \\
\hline 51 & Aporosa cardiosperma (Gaertn.) Merr. & Medicine (Nambiar et al., 1985) \\
\hline 52 & Breynia retusa (Dennst.) Alston & Medicine (Ambasta, 1986) \\
\hline 53 & Briedelia retusa (L.) A. Juss. & $\begin{array}{l}\text { Medicine (Parrota, 2001) } \\
\text { Timber (Ambasta, 1986) }\end{array}$ \\
\hline 54 & Briedelia stipularis (L.) Blume & Medicine (Nambiar et al., 1985) \\
\hline 55 & Euphorbia hirta $\mathrm{L}$. & Medicine (Kirtikar \& Basu, 1918) \\
\hline 56 & Euphorbia thymifolia $\mathrm{L}$. & Medicine (Kirtikar \& Basu, 1918) \\
\hline 57 & Flueggea virosa (Roxb. ex Willd.) Voigt & \\
\hline 58 & Macaranga peltata (Roxb.) Muell.-Arg. & \\
\hline 59 & $\begin{array}{l}\text { Manihot carthaginensis } \\
\text { ssp. glaziovii (Muell-.Arg.) Allem }\end{array}$ & \\
\hline 60 & Micrococca mercurialis (L.) Benth. & \\
\hline 61 & Microstachys chamaelea (L.) Muell.-Arg. & \\
\hline 62 & Phyllanthus airy-shawii Brunel \& Roux & \\
\hline 63 & Phyllanthus amarus Schum. \& Thonn. & Medicine (Nambiar et al., 1985) \\
\hline \multirow[t]{3}{*}{64} & Phyllanthus myrtifolius Moon & Ornamental \\
\hline & 22. FABACEAE & \\
\hline & Subfamily Papilionoideae & \\
\hline 65 & Abrus precatorius L. & Medicine (Nambiar et al., 1985) \\
\hline 66 & Abrus pulchellus Wall. ex Thw. & \\
\hline 67 & Dalbergia lanceolaria L. f. & Timber (Ambasta, 1986) \\
\hline 68 & Dalbergia latifolia Roxb. & Timber (Ambasta, 1986) \\
\hline 69 & Desmodium motorium (Houtt.) Merr. & Medicine (Nambiar et al., 1985) \\
\hline 70 & Desmodium triflorum (L.) DC. & Medicine (Kirtikar \& Basu, 1918) \\
\hline 71 & Pterocarpus marsupium Roxb. & $\begin{array}{l}\text { Medicine (Kirtikar \& Basu, 1918) } \\
\text { Timber (Ambasta, 1986) }\end{array}$ \\
\hline 72 & Pueraria phaseoloides (Roxb.) Benth. & \\
\hline 73 & Spatholobus parviflorus (Roxb. ex DC.) O. Ktze. & Medicine (Nambiar et al., 1985) \\
\hline \multirow[t]{2}{*}{74} & Vigna dalzelliana (O. Kzte.) Verdc. & Food (Ambasta, 1986) \\
\hline & Subfamily Caesalpinioideae & \\
\hline \multirow[t]{2}{*}{75} & Senna alata (L.) Roxb. & \\
\hline & Subfamily Mimosoideae & \\
\hline 76 & Acacia pennata (L.) Willd. & Medicine (Kirtikar \& Basu, 1918) \\
\hline 77 & Albizia odoratissima (L. f.) Benth. & Medicine (Nambiar et al., 1985) \\
\hline 78 & Mimosa pudica $\mathrm{L}$. & Medicine (Kirtikar \& Basu, 1918) \\
\hline \multirow[t]{2}{*}{79} & Paraserianthes falcataria (L.) Neils. & \\
\hline & 23. LAMIACEAE & \\
\hline 80 & Anisochilus carnosus (L. f.) Wall. ex Benth. & Medicine (Nambiar et al., 1985) \\
\hline \multirow[t]{2}{*}{81} & Leucas aspera (Willd.) Link & Medicine (Nambiar et al., 1985) \\
\hline & 24. LAURACEAE & \\
\hline 82 & Cinnamomum malabatrum (Burm. f.) Blume & \\
\hline \multirow[t]{2}{*}{83} & Litsea coriacea (Heyne ex Meisner) Hook. f. & \\
\hline & 25. LOGANIACEAE & \\
\hline 84 & Fagraea ceilanica Thunb. & \\
\hline 85 & Strychnos minor Dennst. & \\
\hline \multirow[t]{2}{*}{86} & Strychnos nux-vomica L. & Medicine (Nambiar et al., 1985) \\
\hline & 26. MALVACEAE & \\
\hline 87 & Hibiscus rosa-sinensis L. var. rosa-sinensis & Ornamental \\
\hline 88 & Sida alnifolia $\mathrm{L}$. & Medicine (Nambiar et al., 1985) \\
\hline
\end{tabular}


Anoop P. Balan et al.

\begin{tabular}{|c|c|c|}
\hline 89 & Urena lobata L. ssp. lobata & Medicine (Kirtikar \& Basu, 1918) \\
\hline & 27. MELASTOMACEAE & \\
\hline 90 & Clidemia hirta (L.) D. Don & \\
\hline 91 & Melastoma malabathricum L. & Medicine (Nambiar et al., 1985) \\
\hline 92 & Memecylon umbellatum Burm.f. & $\begin{array}{l}\text { Medicine } \\
\text { (Nambiar et al., 1985) }\end{array}$ \\
\hline 93 & Osbeckia virgata D. Don ex Wight \& Arn. & \\
\hline \multirow[t]{2}{*}{94} & Sonerila wallichii Bennett & \\
\hline & 28. MELIACEAE & \\
\hline \multirow[t]{2}{*}{95} & Naregamia alata Wight \& Arn. & Medicine (Nambiar et al., 1985) \\
\hline & 29. MENISPERMACEAE & \\
\hline 96 & Cyclea peltata (Lam.) Hook. f. \& Thoms. & Medicine (Vijaya Raghavan, 2011) \\
\hline \multirow[t]{2}{*}{97} & Diploclisia glaucescens (Blume)Diels & Medicine (Ambasta, 1986) \\
\hline & 30. MORACEAE & \\
\hline 98 & Artocarpus heterophyllus Lam. & $\begin{array}{l}\text { Medicine (Kirtikar \& Basu, 1918) } \\
\text { Food, Timber } \\
\text { (Ambasta, 1986) }\end{array}$ \\
\hline 99 & Artocarpus hirsutus Lam. & Medicine (Nambiar et al., 1985) \\
\hline 100 & Ficus arnottiana (Miq.) Miq. & Medicine (Nambiar et al., 1985) \\
\hline \multirow[t]{2}{*}{101} & Ficus religiosa L. & \\
\hline & 31. MYRSINACEAE & \\
\hline \multirow[t]{2}{*}{102} & Embelia tsjeriam-cottam (Roem. \& Schult.) DC. & Medicine (Nambiar et al., 1985) \\
\hline & 32. OCHNACEAE & \\
\hline \multirow[t]{2}{*}{103} & Ochna obtusata DC. & \\
\hline & 33. OLEACEAE & \\
\hline 104 & Jasminum coarctatum Roxb. & Medicine (Nambiar et al., 1985) \\
\hline \multirow[t]{2}{*}{105} & Olea dioica Roxb. & $\begin{array}{l}\text { Medicine (Kirtikar \& Basu, 1918) } \\
\text { Timber (Watt, 1885) }\end{array}$ \\
\hline & 34. ORCHIDACEAE & \\
\hline 106 & Bulbophyllum sterile (Lam.) Suresh & \\
\hline 107 & Dendrobium barbatulum Lindl. & \\
\hline 108 & Habenaria longicornu Lindl. & \\
\hline 109 & Pholidota imbricata Hook. & \\
\hline \multirow[t]{2}{*}{110} & Seidenfia rheedei (Sw.) Szlach. & \\
\hline & 35. OXALIDACEAE & \\
\hline 111 & Biophytum sensitivum (L.) DC. & Medicine (Kirtikar \& Basu, 1918) \\
\hline \multirow[t]{2}{*}{112} & Oxalis corniculata $\mathrm{L}$. & Medicine (Kirtikar \& Basu, 1918) \\
\hline & 36. PERIPLOCACEAE & \\
\hline \multirow[t]{2}{*}{113} & Hemidesmus indicus (L.) R. Br. & Medicine (Nambiar et al., 1985) \\
\hline & 37. PIPERACEAE & \\
\hline 114 & Peperomia pellucida (L.) Kunth & \\
\hline \multirow[t]{2}{*}{115} & Piper trioicum Roxb. & Medicine (Nambiar et al., 1985) \\
\hline & 38. POACEAE & \\
\hline 116 & Alloteropsis cimicina (L.) Stapf & \\
\hline 117 & Arthraxon hispidus (Thunb.) Makino & Fodder (Ambasta, 1986) \\
\hline 118 & Axonopus compressus (Sw.) P. Beauv. & \\
\hline 119 & Digitaria ciliaris (Retz.) Koeler & \\
\hline 120 & Eragrostis unioloides (Retz.) Nees ex Steud. & Fodder (Rama Rao, 1914) \\
\hline 121 & Oplismenus compositus (L.) P. Beauv. & \\
\hline \multirow[t]{2}{*}{122} & Pennisetum polystachyon (L.) Schult. & Fodder (Ambasta, 1986) \\
\hline & 39. RHAMNACEAE & \\
\hline \multirow[t]{2}{*}{123} & Ziziphus oenoplia (L.) Mill. & Medicine (Nambiar et al., 1985) \\
\hline & 40. RUBIACEAE & \\
\hline 124 & Canthium angustifolium Roxb. & \\
\hline 125 & Canthium coromandelicum (Burm. f.) Alston & \\
\hline
\end{tabular}


Floristic Diversity of Thevarmala Sacred Grove in Western Ghats, Kerala, India

\begin{tabular}{|c|c|c|}
\hline 126 & $\begin{array}{l}\text { Chassalia curviflora (Wall. ex Kurz) Thw. var. } \\
\text { ophioxyloides (Wall.) Deb \& Krishna }\end{array}$ & Medicine (Rama Rao, 1914) \\
\hline 127 & Coffea arabica $\mathrm{L}$. & $\begin{array}{l}\text { Medicine (Kirtikar \& Basu, 1918) } \\
\text { Food (Ambasta, 1986) }\end{array}$ \\
\hline 128 & Ixora coccinea $\mathrm{L}$. & Medicine (Kirtikar \& Basu, 1918) \\
\hline 129 & Mitracarpus hirtus (L.) DC. & \\
\hline 130 & Mussaenda frondosa $\mathrm{L}$. & Medicine (Kirtikar \& Basu, 1918) \\
\hline 131 & Oldenlandia auricularia (L.) K. Schum. & \\
\hline 132 & Oldenlandia umbellata L. & \\
\hline 133 & Psilanthus travancorensis (Wight \& Arn.) Leroy & \\
\hline 134 & Spermacoce latifolia Aubl. & \\
\hline \multirow[t]{2}{*}{135} & Spermacoce ocymoides Burm.f. & \\
\hline & 41. RUTACEAE & \\
\hline 136 & Aegle marmelos (L.) Correa & Medicine (Nambiar et al., 1985) \\
\hline \multirow[t]{2}{*}{137} & Glycosmis pentaphylla (Retz.) DC. & Medicine (Watt, 1885) \\
\hline & 42. SAPINDACEAE & \\
\hline \multirow[t]{2}{*}{138} & Allophylus cobbe (L.) Raeusch. & Medicine (Ambasta, 1986) \\
\hline & 43. SCROPHULARIACEAE & \\
\hline 139 & Lindernia crustacea (L.) F.v. Muell. & \\
\hline \multirow[t]{2}{*}{140} & Scoparia dulcis L. & Medicine (Kirtikar \& Basu, 1918) \\
\hline & 44. SMILACACEAE & \\
\hline \multirow[t]{2}{*}{141} & Smilax zeylanica $\mathrm{L}$. & Medicine (Ambasta, 1986) \\
\hline & 45. SOLANACEAE & \\
\hline \multirow[t]{2}{*}{142} & Solanum violaceum Ortega & Medicine (Ambasta, 1986) \\
\hline & 46. STERCULIACEAE & \\
\hline 143 & Helicteres isora $\mathrm{L}$. & Medicine (Nambiar et al., 1985) \\
\hline 144 & Pterospermum diversifolium Blume & \\
\hline \multirow[t]{2}{*}{145} & Sterculia villosa Roxb. ex Smith & \\
\hline & 47. URTICACEAE & \\
\hline \multirow[t]{2}{*}{146} & Pouzolzia zeylanica (L.) Bennett & Medicine (Nambiar et al., 1985) \\
\hline & 48. VERBENACEAE & \\
\hline 147 & Clerodendrum infortunatum $\mathrm{L}$. & Medicine (Kirtikar \& Basu, 1918) \\
\hline 148 & Lantana camara $\mathrm{L}$. & Medicine (Kirtikar \& Basu, 1918) \\
\hline 149 & Stachytarpheta jamaicensis (L.) Vahl & Medicine (Parrota, 2001) \\
\hline \multirow[t]{2}{*}{150} & Vitex altissima L. f. & \\
\hline & 49. VITACEAE & \\
\hline \multirow[t]{2}{*}{151} & Cissus discolor Blume & \\
\hline & 50. ZINGIBERACEAE & \\
\hline 152 & Curcuma neilgherrensis Wight & \\
\hline
\end{tabular}

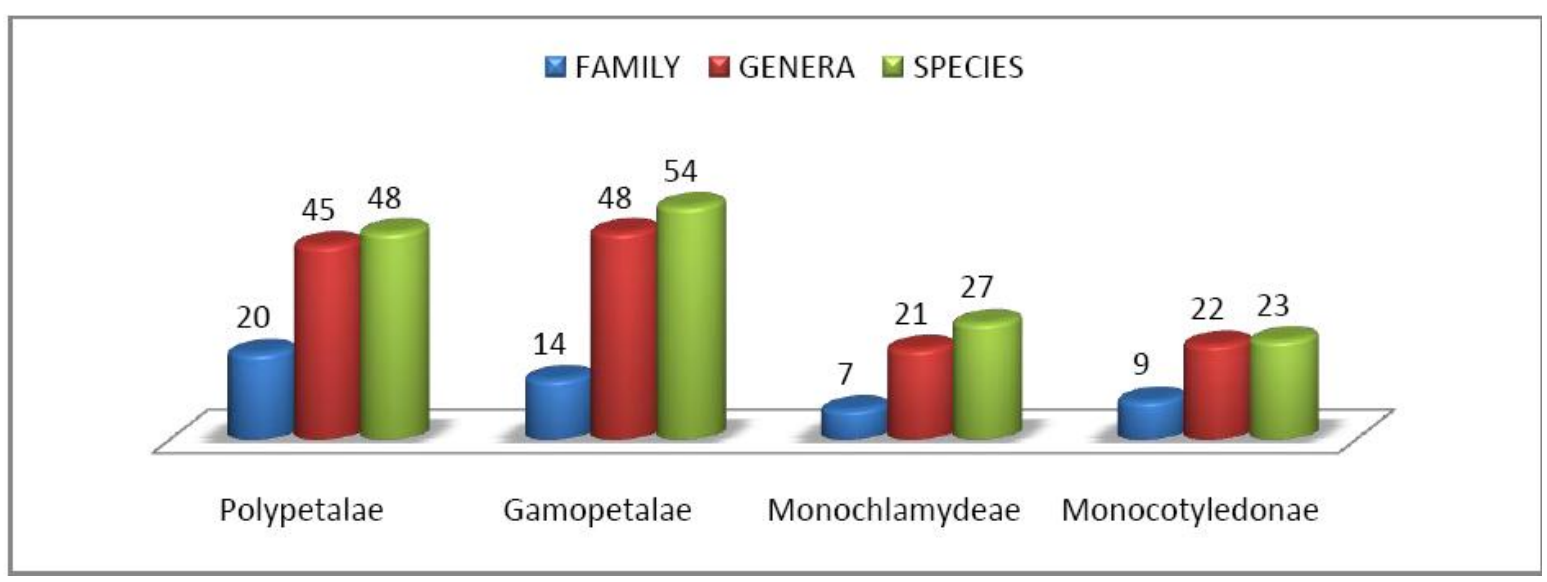

Figure 2. Phytodiversity analysis of Thevarmala sacred grove. 
Among the families, Fabaceae dominates with 15 species in 12 genera followed by Euphorbiaceae (14 species in 10 genera) and Rubiaceae (12 species in 9 genera). As many as 16 families are represented by single species in each (Fig. 3.).

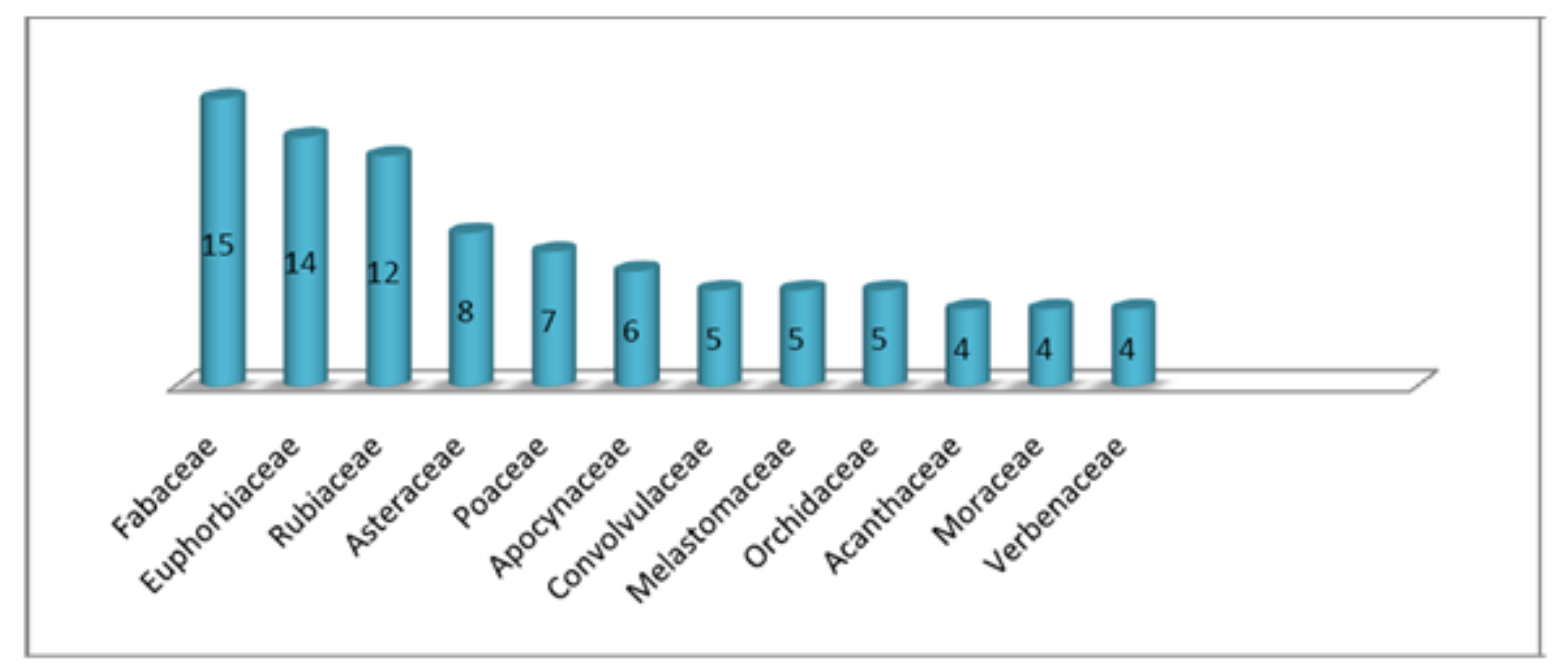

Figure 3. Analysis of dominant Angiosperm families in Thevarmala sacred grove.

The analysis of total life forms present in the sacred grove (Fig. 4.) revealed that shrubs are dominating with 64 species (42\%) followed by herbs with 56 species $(37 \%)$ and trees with 32 species $(21 \%)$. Almost half of the shrubs are climbers. Among the herbs, 3 orchids, namely, Bulbophyllum sterile (Lam.) Suresh, Dendrobium barbatulum Lindl. and Pholidota imbricata Hook. are epiphytes.

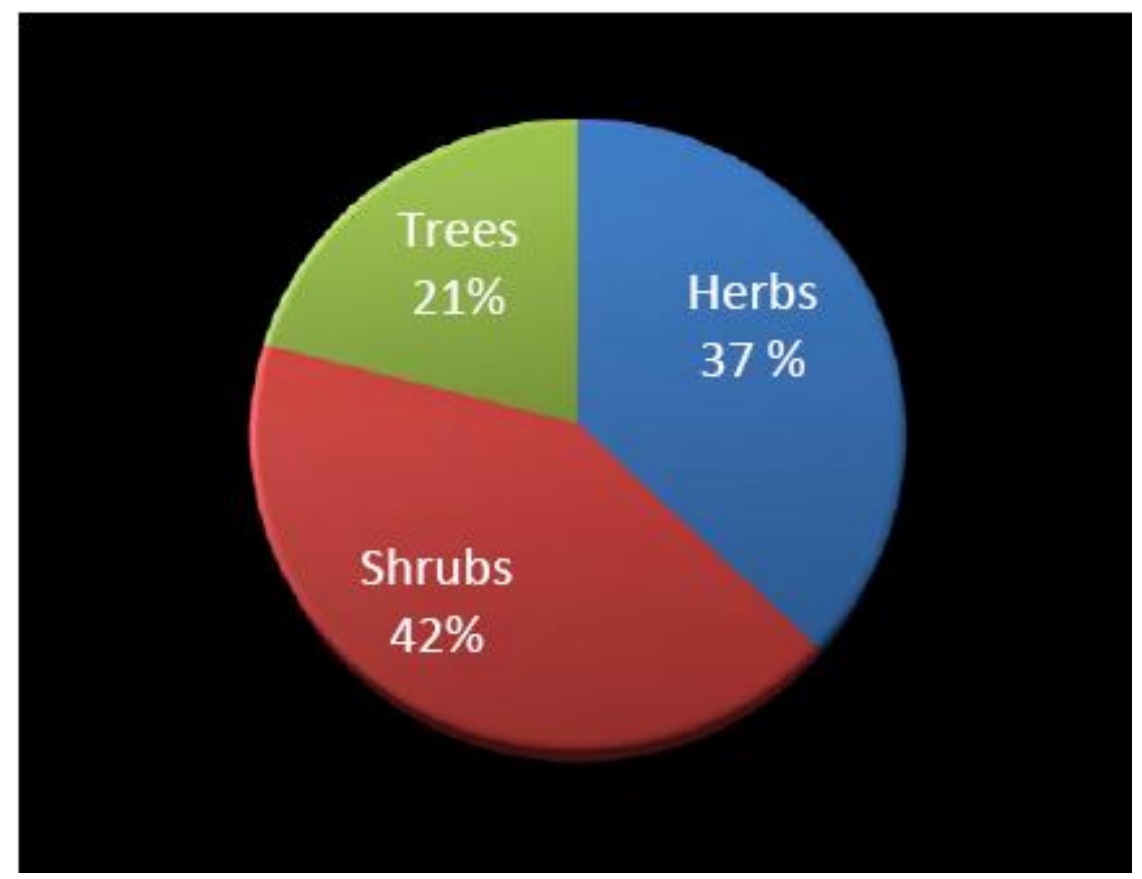

Figure 4. Life form analysis of flowering plants in Thevarmala sacred grove.

\subsection{Endemic Plants}

Endemism encompasses taxonomic units of any rank or taxa which occur in a biogeographical area usually isolated by geographical, ecological or temporal barriers and such species are known as endemic species. Thevarmala sacred grove is located in the Western Ghats, one of the world recognized biodiversity hotspot. Out of the 152 species documented in the present study 18 are endemic to Indian peninsula and majority of them are strictly confined to the Southern Western Ghats (Fig. 5.a-r.). 18 species found only in the Western Ghats-Sri Lanka biodiversity hotspot. Presence of Critically Endangered trees like Vateria indica L., etc. makes the flora of this sacred grove invaluable. 


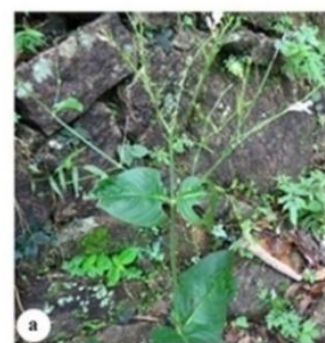

Andrographis atropurpurea

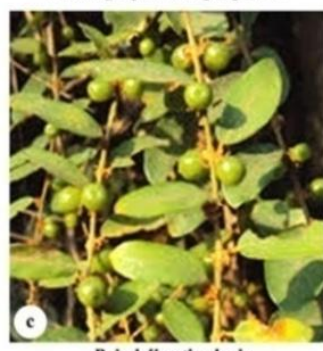

Briedelia stipularis

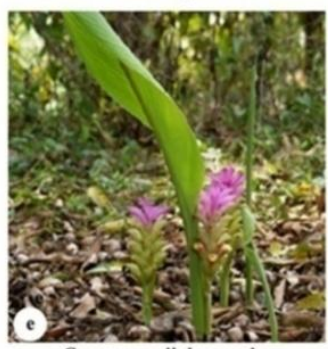

Curcuma neilgherrensis

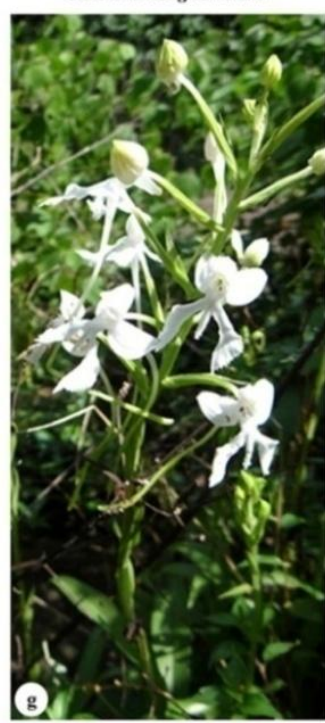

Habenaria longicornu
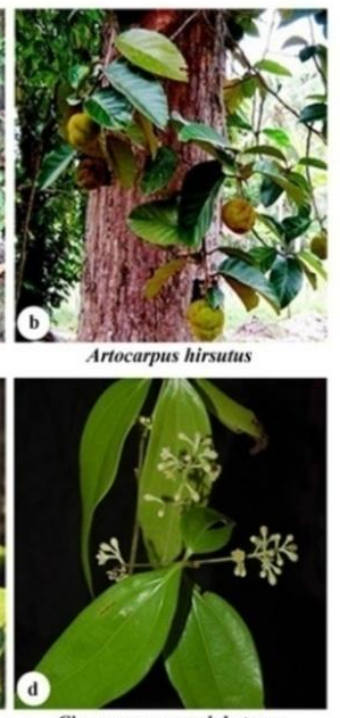

Cinnamomum malabatrum

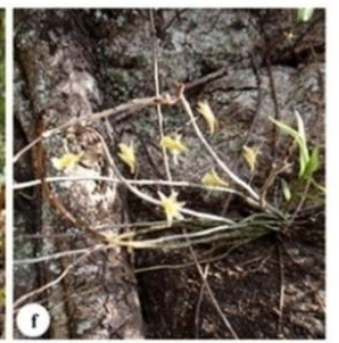

Dendrobium barbatulum

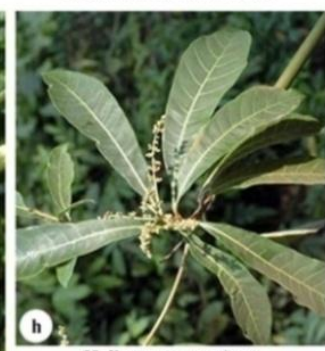

Holigarna arnottiana

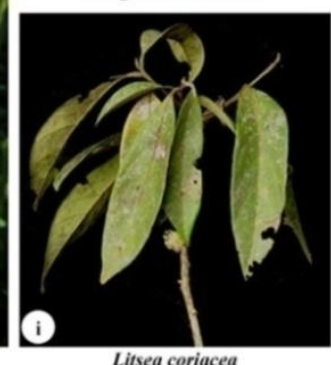

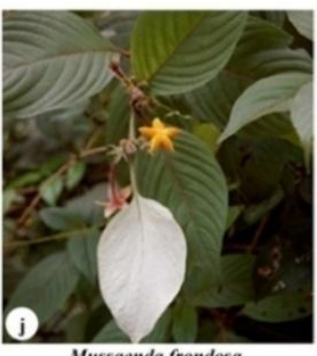

Mussaenda frondosa

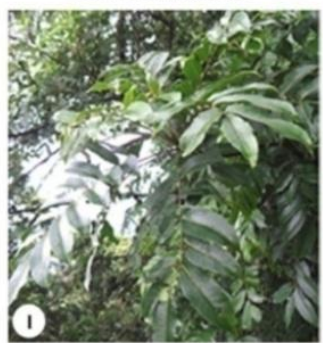

Solenocarpus indicus

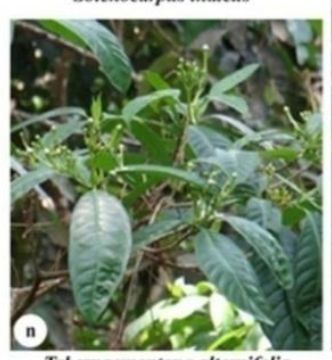

Tabernaemontana alternifolia

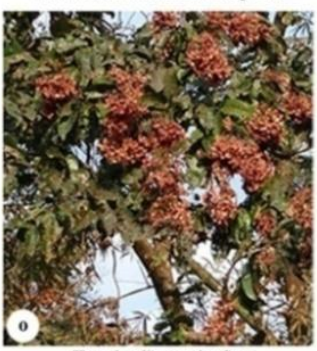

Terminalia paniculata

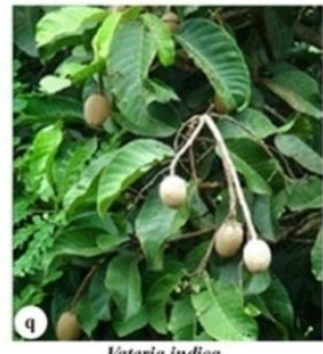

Vateria indica
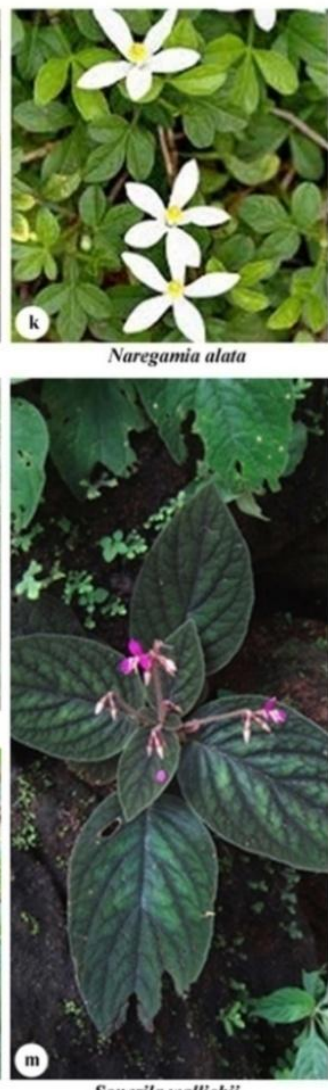

Sonerila wallichii

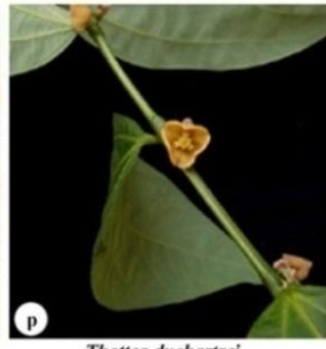

Thottea duchartrei

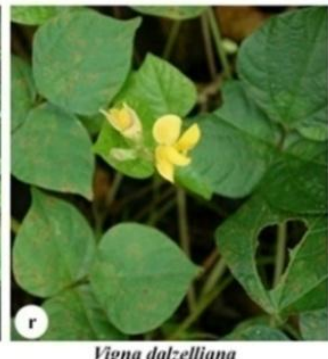

Figure 5. a.-r. Indian endemic plants found in Thevarmala sacred grove.

\subsection{Economically important Plants}

Thevarmala sacred grove is endowed with a large number of medicinally and economically useful plants. About half of the documented plants are having recorded medicinal properties. Anisochilus carnosus (L.f.) Wall. ex Benth., Aristolochia indica L., Cosmostigma racemosum (Roxb.) Wight, Dioscorea oppositifolia L., Embelia tsjeriam-cottam (Roem. \& Schult.) DC., Gymnema sylvestre (Retz.) R. Br. ex Schult., Hemidesmus indicus (L.) R. Br., Holarrhena pubescens (Buch.-Ham.) Wall. ex G. Don, Ichnocarpus frutescens (L.) R. Br., Strychnos nux-vomica L., Vateria indica L., Wrightia tinctoria (Roxb.) R. Br., etc. are some widely used medicinal plants located in this sacred grove. Many plants are economically important as food ( 8 species), timber ( 6 species), and fodder ( 3 species) and as sources of fibres and resins ( 2 species). 


\subsection{Additions to the Flora of Kottayam District}

Thevarmala sacred grove is also home to several plants that are not common in the rest of Kottayam district. Abrus pulchellus Wall. ex. Thw. (Fabaceae), Aganosma cymosa (Roxb.) G. Don (Apocynaceae), Canthium angustifolium Roxb. (Rubiaceae), Canthium coromandelicum (Burm. f.) Alston (Rubiaceae), Calamus rotang L. (Arecaceae), Commelina ensifolia R. Br. (Commelinaceae), Dendrobium barbatulum Lindl. (Orchidaceae), Diploclisia glaucescens (Blume) Diels (Menispermaceae), Embelia tsjeriam-cottam (Roem. \& Schult.) DC. (Myrsinaceae), Habenaria longicornu Lindl. (Orchidaceae), Impatiens flaccida Arn. (Balsaminaceae), Jasminum coarctatum Roxb. (Oleaceae), Justacia diffusa Willd. (Acanthaceae), Phyllanthus airy-shawii Brunel \& Roux (Euphorbiaceae), Schefflera venulosa (Wight \& Arn.) Harms (Araliaceae), Solenocarpus indicus Wight \& Arn. (Anacardiaceae), Sterculia villosa Roxb. ex Smith (Sterculiaceae), Toxocarpus kleinii Wight \& Arn. (Asclepiadaceae) and Thottea duchartrei Sivar., Babu \& Indu (Aristolochiaceae) are new additions to the flora of Kottayam district.

\section{Conclusion}

The present study aimed to study the floristic wealth of Thevarmala sacred grove, Kayyoor in Kottayam district of Kerala state. The study revealed that, the sacred grove is rich in its floristic wealth and is an abode of certain rare endemic as well as medicinally useful plants. A total of 152 species of flowering plants belong to 136 genera in 50 families were collected during the study and the specific and generic diversity present in this comparatively small area of land is exceptional. Among the 152 species documented in the present study, 18 species are Indian endemics and majority of them are very rare and are extremely restricted to the Southern Western Ghats. 19 species reported in this present study is new addition to the Kottayam district. While the adjacent forest areas were all cleared for agriculture and human settlements, this sacred grove is the last refuge for such rare plants in that area.

Similar to all other sacred groves, the sacred religious beliefs and associated taboos helped to prevent the over exploitation of the natural resources present in Thevarmala sacred grove. However, the present status of this sacred grove is rather precarious. Earlier the ' $k a v u$ ' was spreads in 50 acres of land, which has now been reduced to just 15 acres. Human interactions led to the degradation of the natural flora to a certain extent. Invasive alien species like Alternanthera brasiliana ( L. ) Kuntze, Chromolaena odorata (L.) King \& Robins., Lantana camera L., etc. are also a threat to the native flora. Pressure from the ever increasing human population and the agriculture related activities in the adjacent private properties are adversely affecting this serene ecosystem. In this context, necessary actions are urgently needed to protect the invaluable natural wealth of this sacred grove from further degradation. The present scientific documentation of the floristic wealth of this sacred grove will be baseline data for the stakeholders to plan conservation strategies in the future.

\section{ACKNOWLEDGEMENT}

The authors are grateful to the Principal, management and staff of St. Dominic's College, Kanjirapally for providing the necessary facilities and encouragement; Dr. Jojo George, Head of the department of Botany for his constant support; Dr. N. Sasidharan, Kerala Forest Research Institute, Peechi for the help given in the identification of some plants; Mr. Sebastian Antony, St. Berchman's College, Changanassery and Fr. Sebastian Kiliroopparambil for various help. We are also thankful to the authorities of Thevarmala sacred grove for their whole hearted support and necessary help during the field studies.

\section{REFERENCES}

[1] Ambasta, S.P. (ed.), Useful Plants of India, Council of Scientific and Industrial Research Publications, New Delhi (1986).

[2] Chandrashekara, U.M., Conservation and management of sacred groves in Kerala, KFRI Research Report No. 412, Kerala Forest Research Institute, Peechi (2011).

[3] Gamble J.S. and C.E.C. Fischer, The Flora of the Presidency of Madras, Adlard \& Son Ltd., London (1915-1936).

[4] Haritha R. Nair, Binu Thomas, Anish Babu V.B. and Antony V.T., The Role of selected sacred groves in the biodiversity conservation of Kottayam district, Kerala, India, Plant Sciences Feed 5(1), 1-15 (2015). 
[5] Haritha R. Nair, Binu Thomas, Anish Babu V.B., Antony V.T., and M.C. Varghese, Religious important plant from selected sacred groves of Kottayam district, Kerala, India, Botanical Report 3(2), Pp. 1-10 (2014).

[6] Hughes, J.D. and Chandran, M.D.S., Sacred groves around the earth: An overview, In: Ramakrishnan, P.S., Saxena K.G. and Chandrashekara U.M. (eds.) Conserving the sacred for biodiversity Management, Oxford and IBH publishing Co Pvt. Ltd., New Delhi (1998).

[7] Induchoodan, N.C., Ecological Studies of the Sacred Groves of Kerala, Ph. D Thesis, Central University, Pondichery (1998).

[8] Jayarajan, M., Sacred groves of North Malabar, Discussion paper-92, Kerala Research Programme on Local Development (2004).

[9] Kirtikar, K.R. \& B.D. Basu,. Indian Medicinal Plants, Vol.1., Bishen Singh Mahendra Pal Singh, Dehra Dun, 1918 (1975 repr. ed.).

[10] Murugan, K., Ramachandran, V.S., Swarupanadan, K. and Ramesh M., Socio-cultural perspectives to the sacred groves and serpentine worship in Palakkad district, Kerala, Ind. J. Trad. Knowledge 7(3), 455-456 (2008).

[11] Nambiar, V.P.K., N. Sasidharan, C. Renuka and M. Balagopalan, Studies on the Medicinal Plants of Kerala Forests, KFRI Research Report 42, Kerala Forest Research Institute, Peechi (1985).

[12] Nayar, T.S., A. Rasiya Beegam \& M. Sibi, Flowering Plants of the Western Ghats, India, Vol. 1 \& 2, Jawaharlal Nehru Tropical Botanic Garden \& Research Institute, Thiruvananthapuram (2014).

[13] Parrota, J.A., Healing Plants of Peninsular India, CABI Publishing, Wallingford, UK (2001).

[14] Rama Rao, M., Flowering Plants of Travancore, Govt. Press, Trivandrum (1914).

[15] Sasidharan N., Biodiversity documentation for Kerala, part 6; Flowering plants, Kerala Forest Research Institute, Peechi (2004).

[16] Sreevidhya E.A., Ecological importance of sacred groves in the semi urban landscape of Palakkad, Sahyadri Conservation Series 47, 252-257 (2014).

[17] Vartak, V.D., Observation on rare, imperfectly known and endemic plants in the sacred groves of Western Maharashtra, In: S.K. Jain and R.R. Rao (Eds.), An Assessment of threatened plants of India, Botanical Survey of India, Howrah, Pp. 169-178 (1983).

[18] Vijaya Raghavan, G., Comprehensive Medicinal Plants, Vol.1, Studium Press LLC, Texas, USA (2011).

[19] Watt, G., A dictionary of the Economic Products of India, Vol.1, Periodical Experts, Delhi (1972 -Repr. ed.).

\section{AUTHORS' BIOGRAPHY}

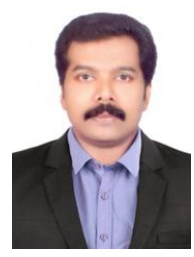

Anoop P. Balan, Sr. Agriculture Demonstrator in Spices Board, Government of India. Actively engaged in angiosperm taxonomic studies in south India especially in the family Leguminosae. Published more than 15 research papers in reputed journals in the concerned field including few plant discoveries.

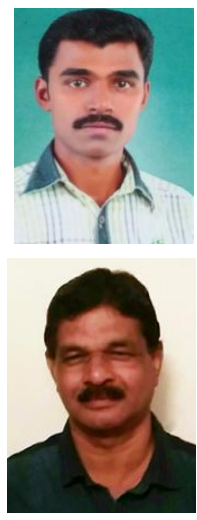

Bibin Thomas, Post Graduate student in the department of Botany, St. Dominic's college, Kanjirappally. Interested in the field of plant taxonomy.

Joseph Michael, Assistant Professor in the department of Botany, St. Dominic's college, Kanjirapally. 